\title{
Quasi-cylindrical approximation to the swirling flow in an atomizer chamber
}

\author{
F. J. Higuera ${ }^{1} \uparrow$ and A. Pereña ${ }^{1}$ \\ ${ }^{1}$ Escuela Técnica Superior de Ingenieros Aeronáuticos. Universidad Politécnica de Madrid, \\ Plaza Cardenal Cisneros 3, 28040 Madrid, Spain
}

(Received 25 June 2014: revised 8 September 2014; accepted 16 September 2014)

A quasi-cylindrical approximation is used to analyse the axisymmetric swirling flow of a liquid with a hollow air core in the chamber of a pressure swirl atomizer. The liquid is injected into the chamber with an azimuthal velocity component through a number of slots at the periphery of one end of the chamber, and flows out as an annular sheet through a central orifice at the other end, following a conical convergence of the chamber wall. An effective inlet condition is used to model the effects of the slots and the boundary layer that develops at the nearby endwall of the chamber. An analysis is presented of the structure of the liquid sheet at the end of the exit orifice, where the flow becomes critical in the sense that upstream propagation of long-wave perturbations ceases to be possible. This analysis leads to a boundary condition at the end of the orifice that is an extension of the condition of maximum flux used with irrotational models of the flow. As is well known, the radial pressure gradient induced by the swirling flow in the bulk of the chamber causes the overpressure that drives the liquid towards the exit orifice, and also leads to Ekman pumping in the boundary layers of reduced azimuthal velocity at the convergent wall of the chamber and at the wall opposite to the exit orifice. The numerical results confirm the important role played by the boundary layers. They make the thickness of the liquid sheet at the end of the orifice larger than predicted by irrotational models, and at the same time tend to decrease the overpressure required to pass a given flow rate through the chamber, because the large axial velocity in the boundary layers takes care of part of the flow rate. The thickness of the boundary layers increases when the atomizer constant (the inverse of a swirl number, proportional to the flow rate scaled with the radius of the exit orifice and the circulation around the air core) decreases. A minimum value of this parameter is found below which the layer of reduced azimuthal velocity around the air core prevents the pressure from increasing and steadily driving the flow through the exit orifice. The effects of other parameters not accounted for by irrotational models are also analysed in terms of their influence on the boundary layers.

Key words: aerosols/atomization, boundary layers, drops and bubbles 


\section{Introduction}

Pressure swirl atomizers are one of the most common types of atomizer used in a large variety of industrial applications, such as oil-fired furnaces and gas turbines, agricultural spraying machinery, fire suppression and spray drying, among others. These atomizers generate a conical sheet of rotating liquid that rapidly breaks into ligaments and then drops. To generate this sheet, liquid is injected into an axisymmetric chamber through tangential slots that induce a swirling flow in the chamber, in the form of a hollow vortex with an air core extending along the axis of the chamber. This flow converges towards a central exit orifice in a process that intensifies the rotation of the liquid, which emerges from the chamber as an annular rotating sheet that rapidly becomes conical when its radial motion ceases to be restricted by the chamber wall.

Important characteristics of the atomizer include the thickness of the annular sheet emerging from the chamber, the angle of the conical sheet that develops at some distance downstream, and the discharge coefficient, which is the flow rate of liquid fed into the atomizer scaled with the product of the area of the exit orifice and the square root of twice the overpressure in the chamber divided by the density of the liquid. All these characteristics depend on the swirling flow in the chamber, which is fairly complex. However, much information has been gathered using irrotational models of this flow (Abramovich 1944; Novikov 1948; Taylor 1948; Giffen \& Muraszew 1953; Nieuwkamp 1985) - see Chinn (2009) for a review. Differing in other respects, these models neglect the radial velocity of the liquid and use the condition that the flow at the end of the exit orifice is critical, in the sense that its axial velocity coincides with the velocity of long waves propagating in the swirling sheet (Binnie \& Hookings 1948; Binnie 1949). Irrotational models give the characteristics of the atomizer mentioned above as functions of a single dimensionless parameter, the atomizer constant, which is the cross-sectional area of the slots through which the liquid is injected into the chamber scaled with the product of the chamber and orifice diameters. Numerical computations of the irrotational, free boundary problem determining the flow and the liquid-air interfaces without additional assumptions have been carried out by Park \& Heister (2006).

Boundary layers develop at the walls of the chamber. The radial pressure gradient that accompanies the swirling motion in the bulk of the chamber is imposed on these layers, but, since the azimuthal velocity falls to zero at the walls, the reduced centripetal acceleration in the boundary layers cannot balance the imposed pressure gradient when the wall is not parallel to the axis of the chamber. The unbalanced pressure gradient (Ekman pumping) drives the liquid in the boundary layer at the convergent wall of the chamber towards the exit orifice, and the liquid in the boundary layer at the wall opposite to the orifice towards the air core. This liquid turns on reaching the liquid-air interface and flows towards the orifice surrounding the air core. As noted by Taylor (1948), the boundary layers that appear by these means at either side of the annular sheet emerging from the orifice may cover a substantial part of the sheet in typical conditions, which limits the validity of irrotational models. The boundary layers have been analysed using integral methods (Binnie \& Harris 1950; Taylor 1950; Cooke 1952), experiments (Binnie, Hookings \& Kamel 1957), and numerical and asymptotic techniques (Burggraf, Stewartson \& Belcher 1971; Bloor \& Ingham 1977; Dumouchel et al. 1992).

A variety of experimental and numerical techniques have been used to characterize the internal flow in atomizer chambers. Experiments with various geometrical configurations, liquid properties and injection pressures and/or flow rates (Dombrowski 
\& Hasson 1969; Rizk \& Lefebvre 1985; Horvay \& Leuckel 1986; Suyari \& Lefebvre 1986; Kim et al. 2009) have clarified the effects of these parameters on the characteristics of the atomizer, allowing one to assess and to extend the predictions of irrotational theories (Lefebvre 1989). Large-scale models made of transparent materials have allowed optical access and measurements at increased spatial resolution with laser Doppler velocimetry, particle image velocimetry, laser-induced fluorescence and high-speed video techniques (Horvay \& Leuckel 1986; Holtzclaw et al. 1997; Wang et al. 1999; Donjat et al. 2003). These experiments have revealed the complexity of the flow in the chamber, which includes regions of very different axial and azimuthal velocities, recirculation, non-steadiness, lack of axisymmetry and waves propagating on the liquid-air interface. On the numerical side, Yule \& Chinn (2000) computed the internal flow in chambers that reproduce the large-scale models used in their own experiments and in those of Horvay \& Leuckel (1986). Using an approximate method to locate the air core, these computations show the presence of Görtler vortices near the cylindrical wall of the chamber, when its length is larger than a certain value, and display the possible paths of the axial flow, adjacent to the boundary layers at the wall and around the air core. Jeng, Jog \& Benjamin (1998) computed the axisymmetric flow and the liquid-air interface in an atomizer chamber, determining the characteristics of the liquid sheet that emerges from the exit orifice. Comparisons were made with predictions of irrotational theories and correlations derived by Rizk \& Lefebvre (1985). In two follow-up works, Sakman et al. (2000) and Xue et al. (2004) numerically explored and rationalized the dependence of the atomizer characteristics on geometrical parameters not accounted for by irrotational models. The effects of various geometrical parameters and of the Reynolds number on the size of the air core and other characteristics of the atomizer have also been investigated by Halder, Dash \& Som (2003) and Nouri-Borujerdi \& Kebriaee (2012), while Steinthorsson \& Lee (2000), Hansen et al. (2002), Maatje, von Lavante \& Albina (2002), Madsen, Hjertager \& Solberg (2002) and von Lavante, Maatje \& Albina (2002) have carried out three-dimensional and non-stationary flow computations.

In this paper, the quasi-cylindrical approximation for viscous swirling flows slowly varying in the axial direction is used to describe the internal flow in an atomizer chamber. The nature and limitations of this approximation are discussed, and a critical flow condition at the end of the orifice is proposed that is a direct extension of the condition used with irrotational models. The resulting problem is solved numerically. The numerical results show that the quasi-cylindrical approximation captures many features of the flow in the chamber at a fraction of the cost of a full Navier-Stokes simulation. The discharge coefficient, the thickness of the annular sheet at the end of the orifice, and an approximate value of the angle of the conical sheet are computed as functions of the atomizer constant and other geometrical parameters. The results are in good agreement with previous simulations and experiments, and the simplicity of the quasi-cylindrical approximation allows simple explanations of the observed trends.

\section{Formulation}

Figure 1 is a sketch of the axisymmetric chamber of a pressure swirl atomizer, of radius $R_{w}(x)$, where $x$ is the axial distance along the chamber. The liquid is injected into the chamber at a constant flow rate $Q$ through tangential slots located at the periphery near the left-hand end of the chamber (not shown in the figure), which induce a high azimuthal velocity, and discharges through a central orifice of radius $R_{0}$ at the other end, following a funnel-shaped convergence. As a consequence 


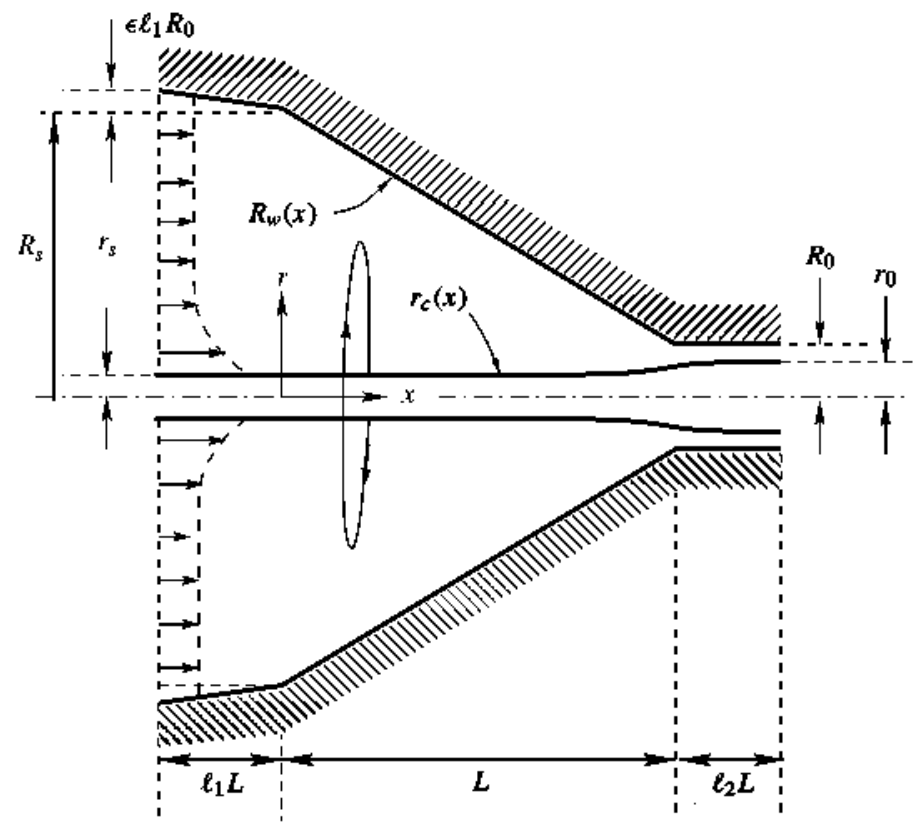

FIGURE 1. Definition sketch.

of the swirling motion of the liquid, an air core of unknown radius $r_{c}(x)$ at the pressure of the atmosphere where the liquid discharges extends along the axis of the chamber, while the pressure increases with radial distance from the liquid-air interface. This overpressure drives the flow towards the discharge orifice, and the azimuthal velocity of the liquid increases on approaching the orifice owing to conservation of angular momentum in this convergent flow. The liquid emerges from the chamber as an annular sheet that tends to becone conical downstream of the chamber and eventually breaks up into drops.

\subsection{Irrotational flow approximation}

Irrotational approximations to the complex flow in an atomizer chamber have been pioneered by Taylor (1948) and Giffen \& Muraszew (1953). Leaving out the immediate neighbourhood of the injection slots, the irrotational flow is assumed to be axisymmetric. The azimuthal velocity of the liquid is then that of a potential vortex, $w=\Gamma / r$, where $r$ is the distance to the axis of the chamber and $2 \pi \Gamma$ is the circulation around any closed curve surrounding the air core. If this expression for the azimuthal velocity is extended to the outlet of the tangential slots (not shown in figure 1), of total cross-sectional area $A_{s}$ at a distance $R_{s}$ from the axis, where the velocity of the liquid is $Q / A_{s}$, then the relation $\Gamma=Q R_{s} / A_{s}$ is obtained. The axial and radial components of the velocity are $u=\partial \varphi / \partial x$ and $v=\partial \varphi / \partial r$, respectively, where the velocity potential $\varphi$ satisfies Laplace's equation $\nabla^{2} \varphi=0$ with Neumann conditions $n \cdot \nabla \varphi=0$ at the chamber walls and at the stationary liquid-air interfaces, and $n \cdot \nabla \varphi=-Q / A_{i}$ at the effective inlet surface of area $A_{i}$ (see (2.11) below). Here $\boldsymbol{n}$ is the unit normal to each boundary. The liquid-air interface $r=r_{c}(x)$, as well as the two surfaces of the liquid sheet outside of the chamber, must be determined as part of the solution using the additional constant-pressure condition $u^{2}+v^{2}=\Gamma^{2}\left(r_{s}^{-2}-r^{-2}\right)$, 
obtained from Bernoulli's equation, with $r_{s}$ denoting the unknown radius of the air core at the far end of the chamber ( $s$ o that the overpressure in the chamber far from the air core is $(\rho / 2)\left(\Gamma / r_{s}\right)^{2}$, where $\rho$ is the density of the liquid).

The flow around the orifice can be computed for large values of $R_{s} / R_{0}$ by replacing the inlet condition above by an asymptotic far-field condition. For a conical chamber wall with half-angle $\beta$, this condition is $|\nabla \varphi| \sim Q /\left[2 \pi(1-\cos \beta)\left(x^{2}+r^{2}\right)\right]$ for $\left(x^{2}+r^{2}\right) \gg R_{0}^{2}$. Scaling distances with $R_{0}$ and velocities with $\Gamma / R_{0}$, the local solution can be seen to depend only on the shape of the chamber wall around the orifice and the dimensionless flow rate $Q / \Gamma R_{0}=A_{s} / R_{s} R_{0}$, where the expression for $\Gamma$ in the previous paragraph has been used to write the last equality. This quantity is four times the atomizer constant, $\Delta=A_{s} /\left(4 R_{s} R_{0}\right)$, a parameter often used in the literature on pressure swirl atomizers (Giffen \& Muraszew 1953).

The local problem can be easily solved in the two asymptotic limits $\left(1-r_{s} / R_{0}\right) \ll 1$ and $r_{s} / R_{0} \ll 1$. The following simplifications can be used in the first case: (i) the thickness of the liquid sheet is small compared to the radius of the orifice; (ii) the Bernoulli equation at the liquid surface reduces to $a^{2}+v^{2} \approx 2 \Gamma^{2} \delta r / r_{s}^{3}$, where $\delta r=$ $r-r_{s}=O\left(R_{0}-r_{s}\right) \ll R_{n}$, implying that the axial velocity of the liquid in this region is only of order $\left(\Gamma / r_{s}\right)\left(1-r_{s} / R_{0}\right)^{1 / 2}$, small compared to the axial and radial velocities of order $\Gamma / r_{s}$ that are attained outside of the chamber; and (iii) Laplace's equation reduces to the planar form $\partial^{2} \varphi / \partial x^{2}+\partial^{2} \varphi / \partial r^{2} \approx 0$ because the term $r^{-1} \partial \varphi / \partial r$ is small compared to $\partial^{2} \varphi / \partial r^{2}$. The reduced problem is equivalent to that of the flow over a broad-crested planar weir, with $\Gamma^{2} / r_{s}^{3}$ playing the role of the acceleration of gravity. The solution (see e.g. Batchelor 1967) gives the flow rate per unit width of the weir as $Q /\left(2 \pi R_{0}\right)=(2 / 3)^{3 / 2}\left(\Gamma / r_{s}^{3 / 2}\right)\left(R_{0}-r_{s}\right)^{3 / 2}$.

In the asymptotic limit $r_{s} / R_{0} \ll 1$, the overpressure of the liquid in the chamber is nearly uniform at $(\rho / 2)\left(\Gamma / r_{s}\right)^{2}$ for any $r \gg r_{s}$. In a first approximation, the discharge of the liquid through the orifice is not affected by the swirling motion at any distance from the air core that is large compared to $r_{s}$, and it is analogous to the discharge of a pressurized liquid at rest in the chamber. If the contraction coefficient can be taken to be unity, this gives $Q=\pi R_{0}^{2} \Gamma / r_{s}$.

Taylor's (1948) analysis of the flow in the atomizer chamber assumes that, for any value of $r_{s} / R_{0}$ : (i) the flow in an axial section is quasi-unidirectional at the end of the orifice, so that Bernoulli's equation at the liquid surface reduces to $u_{0}^{2}=\Gamma^{2}\left(r_{s}^{-2}-r_{0}^{-2}\right)$, where $u_{0}$ and $r_{0}$ are the liquid velocity and the radius of the air core at the end of the orifice; and (ii) the flow rate $Q=\pi u_{0}\left(R_{0}^{2}-r_{0}^{2}\right)$ has its maximum possible value for a given overpressure in the chamber, $(\rho / 2)\left(\Gamma / r_{s}\right)^{2}$, which is equivalent to the condition that the flow at the end of the orifice is critical, in the sense that $u_{0}$ coincides with the velocity of longitudinal waves in the rotating liquid sheet (Binnie \& Hookings 1948; Binnie 1949). These two conditions determine $u_{0}$ and $r_{0}$, and thus the flow rate, for a given $r_{s}$. In Taylor's notation,

$$
\left.\begin{array}{c}
z \equiv \frac{r_{0}}{R_{0}}=\frac{y}{2}\left[1+\left(1+\frac{8}{y^{2}}\right)^{1 / 2}\right]^{1 / 2}, \quad \frac{u_{0}}{\Gamma / r_{s}}=\left(1-\frac{y^{2}}{z^{2}}\right)^{1 / 2}, \\
c_{d} \equiv \frac{Q}{\pi R_{0}^{2} \Gamma / r_{s}}=\left(1-z^{2}\right)\left(1-\frac{y^{2}}{z^{2}}\right)^{1 / 2},
\end{array}\right\}
$$

where $y=r_{s} / R_{0}$ and $c_{d}$ is the discharge coefficient of the atomizer $\left(c_{d}=4 y \Delta / \pi\right)$. These results coincide with the asymptotic results above in the two limits $y \rightarrow 1$ and $y \rightarrow 0$. 


\subsection{Viscous effects}

Boundary layers exist around the walls of the chamber. As mentioned above, Ekman pumping due to the unbalanced pressure gradient in the boundary layers on the convergent wall and on the wall opposite to the orifice pushes the liquid in these boundary layers tangentially to the walls towards the symmetry axis (see e.g. Binnie \& Harris 1950; Taylor 1950; Cooke 1952; Binnie et al. 1957; Burggraf et al. 1971).

These features of the flow are clearly amenable to a boundary layer type of analysis. However, recirculation in the bulk of the atomizer chamber, which is a prominent feature displayed by experimental visualizations (Binnie et al. 1957; Wang et al. 1999; Donjat et al. 2003) and numerical computations (Jeng et al. 1998; Yule \& Chinn 2000; Nouri-Borujerdi \& Kebriaee 2012) for moderate to small values of the atomizer constant, can hardly be accounted for using a boundary layer approximation. The size of the recirculation region and the direction of circulation of the liquid depend on the inlet conditions. Thus, this direction is opposite in the simulations of Jeng et al. (1998), Yule \& Chinn (2000) and Xue et al. (2004), in which the liquid is injected with a radial velocity component, and in those of Nouri-Borujerdi \& Kebriaee (2012), in which it is injected with an axial velocity component. Nevertheless, such large differences do not seem to have a large effect on the characteristic of the atomizer, probably because the velocity components of the recirculating flow in an axial section of the chamber are small compared to the azimuthal velocity in other regions of the chamber and to the velocity around the orifice. These results suggest that a boundary layer approximation can capture many important elements of the flow in an atomizer chamber, and it can do so at a fraction of the cost of a numerical simulation using the full Navier-Stokes equations.

Another limitation of the boundary layer approximation arises from the centrifugal instability of the swirling flow in the boundary layer at the curved wall of the chamber. As noted by Yule \& Chinn (2000), this instability first develops in the cylindrical region that often precedes the convergence towards the exit orifice, where Ekman pumping is absent and the axial velocity is too small to sweep the perturbations. Here, to prevent this instability, the cylindrical surface will be replaced by a slightly convergent conical surface upstream of the main convergence; see figure 1 .

The quasi-cylindrical approximation for rotating axisymmetric flows will be used. In this approximation, axial derivatives are neglected in the viscous terms of the momentum equations, and the radial momentum equation reduces to a balance of pressure force and the radial acceleration due to the swirl. In cylindrical coordinates, scaling axial distance $(x)$ with the length $L$ of the convergent region, radial distance $(r)$ with the radius $R_{0}$ of the orifice, axial and azimuthal velocities $(u$ and $w)$ with $\Gamma / R_{0}$, radial velocity $(v)$ with $\Gamma / L$, and the excess of pressure above the atmospheric pressure with $\rho \Gamma^{2} / R_{0}^{2}$, and denoting dimensionless variables in the rest of the paper with the same symbols used before for their dimensional counterparts, the governing equations become

$$
\begin{gathered}
\frac{\partial u}{\partial x}+\frac{1}{r} \frac{\partial}{\partial r}(r v)=0, \\
u \frac{\partial u}{\partial x}+v \frac{\partial u}{\partial r}=-\frac{\partial p}{\partial x}+\frac{1}{R e} \frac{1}{r} \frac{\partial}{\partial r}\left(r \frac{\partial u}{\partial r}\right), \\
\frac{\partial p}{\partial r}=\frac{w^{2}}{r}, \\
u \frac{\partial}{\partial x}(r w)+v \frac{\partial}{\partial r}(r w)=\frac{1}{R e} r \frac{\partial}{\partial r}\left(\frac{1}{r} \frac{\partial}{\partial r}(r w)\right),
\end{gathered}
$$


where $R e=(\Gamma / v)\left(R_{0} / L\right)$, with $v$ denoting the kinematic viscosity of the liquid, is an effective Reynolds number.

Boundary conditions at the wall of the chamber, $r=R_{w}(x)$, and at the liquid-air surface, $r=r_{c}(x)$, which is to be found as part of the solution, are

$$
\begin{array}{ll}
r=R_{w}(x) ; & u=v=w=0 . \\
r=r_{c}(x) ; & v-u \frac{\mathrm{d} r_{c}}{\mathbf{d} x}=p=\frac{\partial u}{\partial r}=\frac{\partial}{\partial r}\left(\frac{w}{r}\right)=0 .
\end{array}
$$

Here, pressure variations in the air and viscous stresses of the air on the liquid surface, as well as surface tension, are neglected.

For definiteness, the region of the chamber where (2.2)-(2.5) are applied will be assumed to consist of a weakly convergent region of dimensionless length $\ell_{1}$ (scaled with $L$ ) and end dimensionless radius $R_{s}$ (scaled with $R_{0}$ ), followed by a stronger conical convergence of unit dimensionless length and end radius, and by a cylindrical region of dimensionless length $\ell_{2}$ (scaled with $L$ ) and unit dimensionless radius. Thus, setting the origin at the beginning of the strong convergence,

$$
R_{w}(x)= \begin{cases}R_{s}-\epsilon x & \text { for }-\ell_{1}<x<0 \\ R_{s}-\left(R_{s}-1\right) x & \text { for } 0<x<1 \\ 1 & \text { for } 1<x<1+\ell_{2}\end{cases}
$$

Here $\epsilon=0$ corresponds to a cylindrical chamber with a conical contraction, while values of $\epsilon=0.1-0.2$ are used in the computations discussed in the following section to prevent the centrifugal instability mentioned above.

Inlet and outlet boundary conditions require some additional discussion. Since the quasi-cylindrical approximation cannot describe the flow around the inlet slots and in the boundary layer on the far end of the chamber, conditions are imposed at $x=-\ell_{1}$ that approximately account for the real flow. These conditions are

$$
x=-\ell_{1}: \quad u=u_{s 0}+u_{j e t}(r), \quad w=\frac{1}{r}-w_{j e t}(x) .
$$

Here $u_{s 0}$ is taken to be a uniform axial velocity, and $u_{j e t}(r)$ and $w_{j e t}(r)$ are jet-like profiles that account for the excess axial velocity and the defect azimuthal velocity around the air core due to the turn around of the boundary layer on the far end of the chamber. These profiles should come from an analysis of that boundary layer, which has been carried out at various levels of detail when the far end is a planar disk and the liquid is fed symmetrically. However, the flow around the far end of the chamber may be very complex for other configurations of the wall and the inlet slots, which has a bearing on the effective inlet conditions (2.9). This is an additional limitation of the quasi-cylindrical approximation. The following approximate profiles will be used here:

$$
u_{j e t}=u_{c} \exp \left[-\frac{\left(r-r_{c}\left(-\ell_{1}\right)\right)^{2}}{\delta^{2}}\right], \quad w_{j e t}=\frac{1}{r} \exp \left[-\frac{\left(r-r_{c}\left(-\ell_{1}\right)\right)^{2}}{\delta^{2}}\right],
$$

with constant $u_{c}=O(1)$ and $\delta=O\left(R e^{-1 / 2}\right)$. Since the dimensionless flow rate $Q$ (scaled with $\Gamma R_{0}$ ) is given, the uniform part of the inlet velocity is

$$
u_{s 0}=\frac{Q-\int_{r_{c}\left(-\ell_{1}\right)}^{R_{w}\left(-\ell_{1}\right)} 2 \pi r u_{j e t} \mathrm{~d} r}{\pi\left[R_{w}\left(-\ell_{1}\right)^{2}-r_{c}\left(-\ell_{1}\right)^{2}\right]} .
$$


A boundary condition is needed at the end of the orifice $\left(x=1+\ell_{2}\right)$ because the quasi-cylindrical model (2.2)-(2.5) is not parabolic, despite the boundary layer approximation. This is related to the fact that the pressure gradient in (2.3) is not given in advance but is part of the solution, which enables the upstream propagation of waves. The boundary condition sought is a direct extension of the condition of maximum flux used in irrotational models. It states that, on approaching the end of the orifice, the flow must accelerate as much as it can, becoming choked in anticipation of the larger acceleration that it undergoes further downstream, where no chamber wall restricts the radial motion of the liquid. The asymptotic structure of the flow for $x \rightarrow 1+\ell_{2}$ when subjected to this condition has been worked out elsewhere for the related problem of a liquid sheet flowing over the edge of a horizontal plate under the action of gravity (Higuera 1994) and is summarized in $\$ 2.3$ for the problem at hand. In essence, the flow variables $\left(u, w, p, r_{c}\right)$ are bounded but $\partial\left(u, w, p, r_{c}\right) / \partial x=$ $O\left[\left(1+\ell_{2}-x\right)^{\lambda-1}\right]$ with $\lambda \approx 0.308$ when $x \rightarrow 1+\ell_{2}$. This singularity is handled by using a strained variable

$$
\xi=\left(1+\ell_{2}-x\right)^{\kappa} \text { with } \kappa<\lambda \text { so that } \frac{\partial}{\partial \xi}\left(u, w, p, r_{c}\right) \rightarrow 0 \text { for } \xi \rightarrow 0 \text {. }
$$

Problem (2.2)-(2.12) contains the eight dimensionless parameters

$$
R e, Q, R_{s}, \ell_{1}, \ell_{2}, \epsilon, u_{c}, \delta \text {, }
$$

which are, respectively, the effective Reynolds number introduced below (2.5), the dimensionless flow rate injected into the chamber, which is related to the atomizer constant by $Q=4 \Delta$, the four geometrical parameters defining the shape of the chamber as sketched in figure 1, and the two parameters modelling the maximum excess axial velocity and the thickness of the high-speed region at the effective inlet.

We note here for reference that the first and last relations in Taylor's solution (2.1) can be written in our dimensionless variables as $2 r_{0}=r_{s}\left[1+\left(1+8 / r_{s}\right)^{1 / 2}\right]^{1 / 2}$ and $4 r_{s} \Delta / \pi=\left(1-r_{0}^{2}\right)\left(1-r_{s}^{2} / r_{0}^{2}\right)^{1 / 2}$. These two relations implicitly determine $r_{s}$ and $r_{0}$ as functions of $\Delta$, and then $u_{0}=\left(1-r_{s}^{2} / r_{0}^{2}\right)^{1 / 2} / r_{s}$ and $c_{d}=4 r_{s} \Delta / \pi$.

For the numerical treatment, the problem is rewritten in terms of the variables $\xi$ and $\zeta=\left(R_{w}-r\right) /\left(R_{w}-r_{c}\right)$ (which satisfies $0<\zeta<1$ ), discretized using finite differences, and solved with a standard iterative technique that amounts to adding time derivatives to the left-hand sides of (2.3) and (2.5).

\subsection{Critical flow at the end of the orifice}

The analysis leading to the results mentioned above (2.12) is as follows. Let $r_{0}<1$ be the limiting value of the air core radius at the end of the orifice, and $u^{*}(r), w^{*}(r)$ and $p^{*}(r)$ be the unknown limiting distributions of axial velocity, azimuthal velocity and pressure, and advance that $u^{*} \approx \alpha(1-r)^{\sigma}$ and $w^{*} \approx \beta(1-r)^{\tau}$ for some $(\sigma, \tau)<1$ when $(1-r) \ll 1$. Under the action of the divergent, self-induced pressure gradient, viscous effects are confined to a sublayer whose thickness tends to zero when $x \rightarrow 1+\ell_{2}$, while the solution in the rest of the liquid sheet is essentially inviscid and of the form

$$
\left.\begin{array}{c}
u=u^{*}(r)+\left(1+\ell_{2}-x\right)^{\lambda} U(r), \quad w=w^{*}(r)+\left(1+\ell_{2}-x\right)^{\lambda} W(r), \\
p=p^{*}(r)+\left(1+\ell_{2}-x\right)^{\lambda} P(r), \\
v=\left(1+\ell_{2}-x\right)^{\lambda-1} V(r), \quad r_{c}=r_{0}+\left(1+\ell_{2}-x\right)^{\lambda} R,
\end{array}\right\}
$$


for some $0<\lambda<1$. Carrying these expansions into (2.2)-(2.5) and (2.7), and collecting the largest terms in powers of $\left(1+\ell_{2}-x\right)$, we find, after some algebra,

$$
\left[\frac{u^{*^{2}}}{r}\left(r \frac{V}{u^{*}}\right)^{\prime}\right]^{\prime}+\frac{\left[\left(r w^{*}\right)^{2}\right]^{\prime}}{r^{3}} \frac{V}{u^{*}}=0, \quad \text { with } \frac{V}{u^{*}}=\lambda R \text { and }\left(r \frac{V}{u^{*}}\right)^{\prime}=\lambda \frac{w^{*^{2}}}{u^{*^{2}}} R \text { at } r=r_{0} \text {. }
$$

A local analysis of this differential equation for $(1-r) \ll 1$ shows that it has two linearly independent solutions of the form $V \sim(1-r)^{\sigma}$ and $V \sim(1-r)^{1-\sigma}$, for which, from the continuity equation (2.2),

$$
U \sim \frac{1}{(1-r)^{1-\sigma}} \quad \text { and } \quad U \sim \frac{1}{(1-r)^{\sigma}} \quad \text { for }(1-r) \ll 1
$$

The flow in the viscous sublayer is as for the gravity-driven sheet analysed in Higuera (1994). The characteristic thickness $\delta_{v}$ of the viscous sublayer and a first relation between the unknown constants $\sigma$ and $\lambda$ can be obtained from the order-of-magnitude balance of inertia, pressure force and viscous force in the axial momentum equation, $u_{v}^{2} /\left(1+\ell_{2}-x\right) \sim \Delta p /\left(1+\ell_{2}-x\right) \sim R e^{-1} u_{v} / \delta_{v}^{2}$, with the characteristic velocity $u_{v} \sim \delta_{v}^{\sigma}$ in order to match $u^{*}(r)$ and $\Delta p=\left(1+\ell_{2}-x\right)^{\lambda} P(1)$. These two conditions give $\delta_{v} \sim\left[\left(1+\ell_{2}-x\right) / R e\right]^{1 /(\sigma+2)}$ and $\lambda=2 \sigma /(\sigma+2)$. In terms of the stream function, $\psi$, the solution in the viscous sublayer is of the form

$$
\psi=-\left(\frac{1+\ell_{2}-x}{\operatorname{Re}}\right)^{(\sigma+1) /(\sigma+2)} g(\eta), \quad \eta=\frac{1-r}{\left(\frac{1+\ell_{2}-x}{\operatorname{Re}}\right)^{1 /(\sigma+2)}},
$$

where $g(\eta)$ satisfies $(\sigma+2) g^{\prime \prime \prime}+\sigma g^{2}-(\sigma+1) g g^{\prime \prime}+2 \sigma R e^{2 \sigma /(\sigma+2)} P(1)=0$ with $g(0)=$ $g^{\prime}(0)=0$ and the matching condition $g^{\prime} \sim \alpha \eta^{\sigma}$ for $\eta \rightarrow \infty$. This problem has a solution only for a special value of $\sigma=\sigma_{c} \approx 0.364$, and matching to the outer solution (2.14) requires that the coefficient of $1 /(1-r)^{1-\sigma}$ in (2.16) be zero. This is a third condition on the solution of the second-order equation in (2.15), which therefore imposes a restriction on $\left(u^{*}, w^{*}\right)$. That $(2.15)$ has a non-trivial solution (with $R \neq 0$ ) amounts to the condition that the speed of upstream-propagating waves becomes zero at the end of the orifice.

In the case of an irrotational flow $\left(u^{*}\right.$ uniform, $\left.w^{*}=1 / r\right),(2.15)$ has a solution with $V(1)=0$ (actually $\left.V=\left(\lambda R / 2^{1 / 2} r_{0} r\right)\left(1-r^{2}\right) /\left(1-r_{0}^{2}\right)^{1 / 2}\right)$ only if $u^{*}=\left(1-r_{0}^{2}\right)^{1 / 2} / 2^{1 / 2} r_{0}^{2}$, which coincides with the velocity of long waves in an annular sheet of swirling liquid.

\section{Results and discussion}

Figure 2 shows some features of the flow in the atomizer for $R e=2000, R_{s}=3$, $\ell_{1}=0, \ell_{2}=0.2, u_{c}=1$ and different values of the atomizer constant $(\Delta=Q / 4)$ and the parameter $\delta$ measuring the upstream thickness of the boundary layer around the air core. Displayed are axial sections of streamtubes (solid) and isobars (dashed). In agreement with known experimental and numerical results, the isobars are nearly cylindrical in the bulk of the chamber, reflecting the dominant effect of the swirling flow whose centripetal acceleration causes the radial variation of pressure via (2.4), while an axial pressure gradient develops around the orifice that pushes the liquid out of the chamber. The axial velocity is small compared to the azimuthal velocity except in the vicinity of the orifice and in the boundary layers around the convergent wall of the chamber and the air core. The radius of the air core is nearly uniform in the interior of the chamber and increases around the orifice, where the pressure decreases. 
(a)

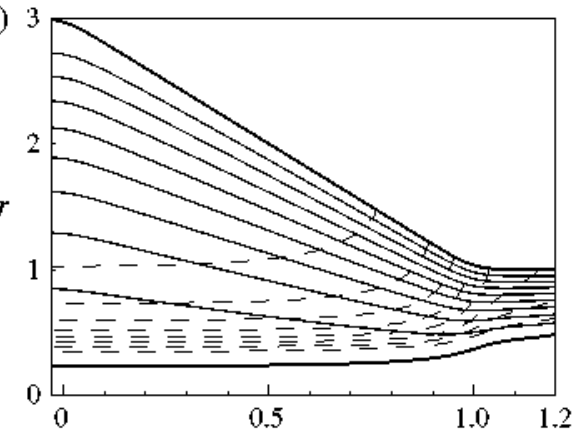

(c)

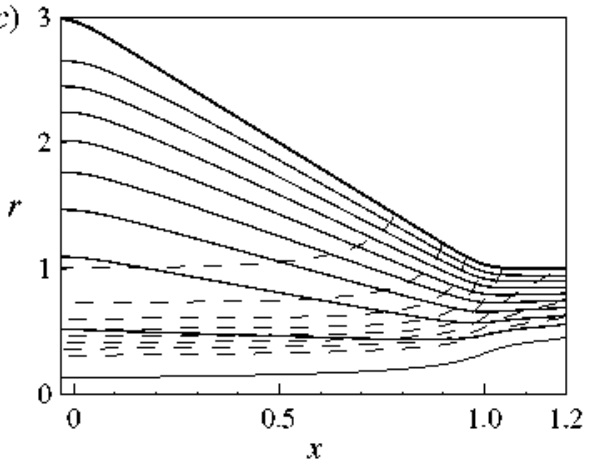

(b)

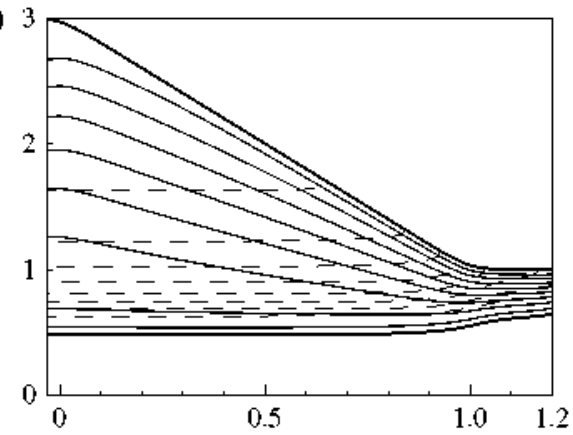

(d)

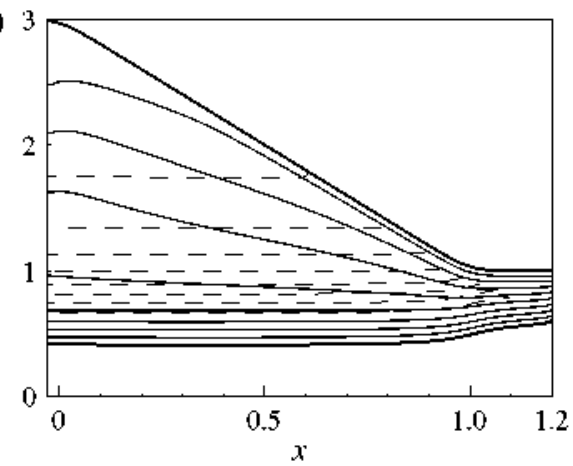

FIGURE 2. (Colour online) Streamlines (solid) and isobars (dashed) for: (a) $\Delta=1.25$, $\delta=0.1$; (b) $\Delta=0.4, \delta=0.1 ;$ (c) $\Delta=1.25, \delta=0.245$; and (d) $\Delta=0.3925, \delta=0.245$. Values of other parameters are $R e=200, R_{s}=3, \ell_{1}=0, \ell_{2}=0.2$ and $u_{c}=1$.

\subsection{Effect of the atomizer constant}

Figure 3(a) shows the discharge coefficient, $c_{d}=Q /\left(2^{1 / 2} \pi p_{s 0}^{1 / 2}\right)$, as a function of the atomizer constant $\Delta$. Here $p_{s 0}$ is the dimensionless chamber overpressure, which is taken as $p_{s 0}=\max _{r}\left[p+\left(u^{2}+w^{2}\right) / 2\right]$ at the inlet section $x=-\ell_{1}$ but outside the boundary layer surrounding the air core. As can be seen, the computed $c_{d}$ is slightly above the inviscid prediction (dotted curve in figure $3 a$ ), which amounts to a chamber overpressure smaller than the inviscid prediction for a given dimensionless flow rate. This is an effect of the excess axial velocity induced by Ekman pumping in the boundary layers, which account for part of the flow rate without requiring any pressure force in the bulk. The fraction of the flow rate that is in the boundary layers increases when the atomizer constant decreases, and the difference between the discharge coefficient and its inviscid prediction then increases. Figure 3(a) also shows that this difference decreases when $\delta$ in (2.10), and thus the thickness of the viscous layer around the air core, decreases. The squares in figure $3(a, d)$ are experimental results from Dombrowski \& Hasson (1969) for $R_{s}=6, \ell_{1}=0, \ell_{2}=0.029$ (filled) and $R_{s}=9, \ell_{1}=0, \ell_{2}=0.018$ (empty). The filled and empty circles are experimental results from Yule \& Widger (1996) and Yule \& Chinn (2000) for different atomizers with $R_{s}=6.54, \ell_{1}=4.94, \ell_{2}=1.18$ and $R_{s}=3.31, \ell_{1}=4.69, \ell_{2}=1.05$, respectively. The diamonds are numerical results from Jeng et al. (1998) for $R_{s}=4.22, \ell_{1}=3.6$, $\ell_{2}=1.1$. Finally, the stars are experimental results from Rizk \& Lefebvre (1985) for $R_{s}=4, \ell_{1}=0.33, \ell_{2}=1.3$. 

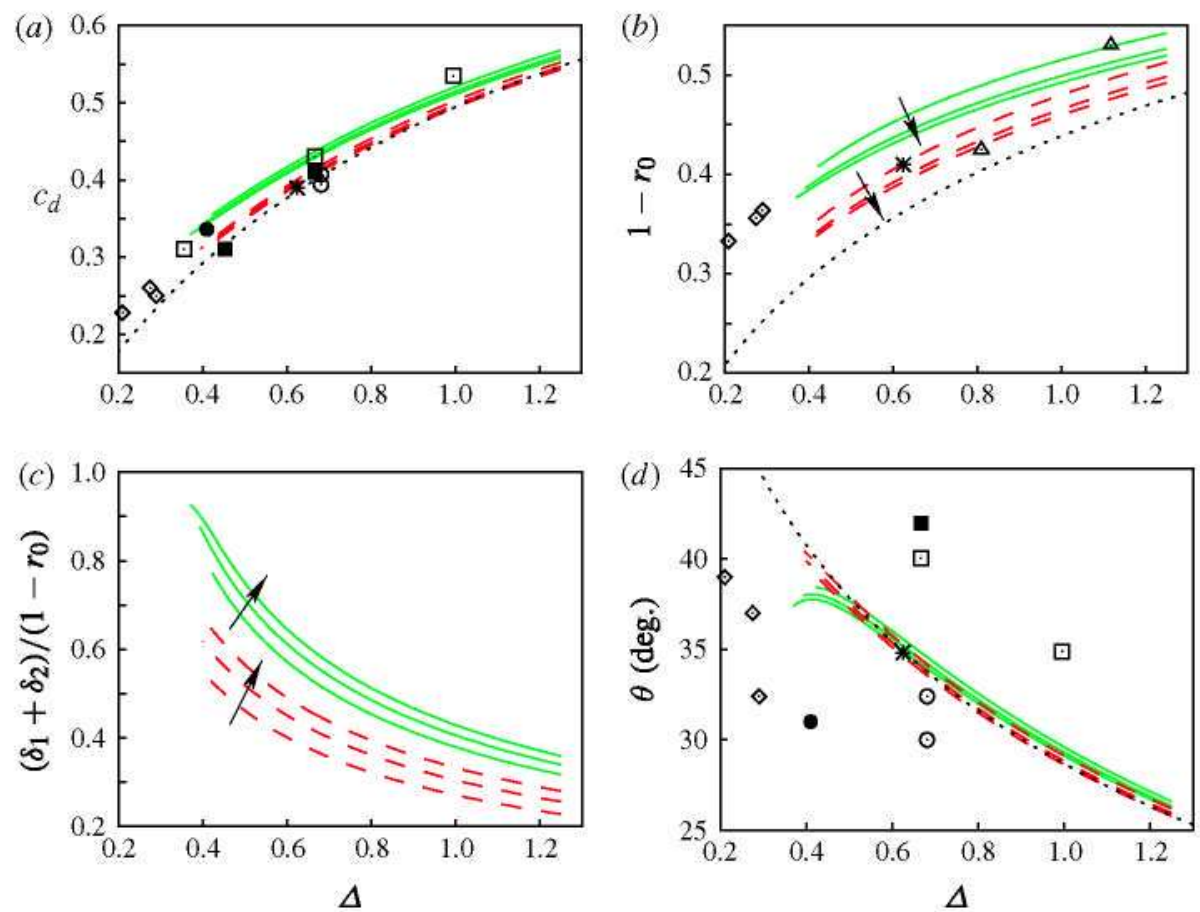

Figure 3. (Colour online) Atomizer characteristics as functions of the atomizer constant $\Delta$ for $\delta=0.245$ (solid curves) and 0.1 (dashed curves), and $\ell_{2}=0,0.2,0.4$, increasing as indicated by the arrows. Values of other parameters are $R e=2000, R_{s}=3, \ell_{1}=0$ and $u_{c}=1$. Dotted curves show the irrotational predictions (2.1). Symbols are experimental and numerical data from Dombrowski \& Hasson (1969) (squares), Yule \& Widger (1996) (filled circles), Yule \& Chinn (2000) (empty circles), Halder et al. (2003) (triangles), Jeng et al. (1998) (diamonds) and Rizk \& Lefebvre (1985) (stars). (a) Discharge coefficient $\left(c_{d}\right)$. (b) Liquid sheet thickness at the end of the orifice $\left(1-r_{0}\right) .(c)$ Sum of the boundary layer thicknesses at the end of the orifice scaled with the local thickness of the liquid sheet $\left(\left(\delta_{1}+\delta_{2}\right) /\left(1-r_{0}\right)\right)$. (d) Half-angle of the conical sheet $(\theta)$.

The computed thickness of the liquid sheet at the end of the orifice is shown in figure $3(b)$ together with the irrotational prediction (dotted), numerical results from Jeng et al. (1998) (diamonds), and experimental results from Rizk \& Lefebvre (1985) (stars) and Halder et al. (2003) (triangles, for $R_{s}=4$ and the two values $\ell_{2}=0.49$ and $\ell_{2}=0.97$ ). The thickness of the sheet is larger than the inviscid prediction due mainly to the boundary layer around the air core. The excess thickness above the inviscid prediction increases with $\delta$. The distances to the solid wall and to the liquidair interface where $r w=0.99$, say $\delta_{1}$ and $\delta_{2}$, are used to define the thicknesses of the boundary layers. The ratio of the sum of these thicknesses to the thickness of the liquid sheet at the end of the orifice is shown in figure $3(\mathrm{c})$ as a function of the atomizer constant. This ratio increases when the atomizer constant decreases, and the boundary layers cover most of the liquid sheet at the smallest value of the atomizer constant for which a solution of (2.2)-(2.12) has been obtained when $\delta=0.245$, in agreement with the estimates of Taylor (1948) and Dumouchel et al. (1992). The presence of the boundary layers also makes the radius of the air core at the bottom of the chamber $\left(r_{s}\right.$, not displayed) smaller than the inviscid prediction. This radius 
increases when $\delta$ decreases, and also when the atomizer constant decreases, which decreases the overpressure in the chamber.

The reduced value of $r w$ in the boundary layer around the air core has a strong effect on the solution because it is in this region of smallest $r$ where most of the radial increase of pressure should occur according to inviscid theory; see (2.4). Thus the concentration of isobars in the vicinity of the air core in figure $2(a)$, for $\delta=0.1$, when the layer of reduced swirl is thin, can be compared with their wider spacing in figure $2(c)$, for $\delta=0.245$, when a thicker region of small $p$ exists around the air core. The thickness of the boundary layer at the end of the orifice and in the interior of the chamber increases when the atomizer constant decreases, which hinders the ability of the atomizer to generate the required overpressure, and eventually leads to the breakdown of the numerical solution. For example, the angle of the isobars to the streamlines in a region around the orifice is higher in figure $2(a, c)$ than in figure $2(b, d)$, suggesting that the pressure force is more efficient at accelerating the liquid in the first case than in the second. The minimum values of the atomizer constant attained in these computations are somewhat larger than in experiments (e.g. Dombrowski \& Hasson 1969), probably due to the small value of the Reynolds number used in the computations.

Conservation of axial and angular momentum of the annular liquid sheet that emerges from the atomizer implies that, as long as air forces can be neglected, the sheet should asymptotically become conical, and the swirl velocity should tend to zero, far from the orifice. For an inviscid liquid, the angle of the cone asymptote can be determined without a detailed analysis of the evolution of the sheet downstream of the chamber (Taylor 1948), but this does not seem to be possible for a real viscous liquid. An approximation derived along the following lines will be used here. First, conservation of axial monentum for a control volume comprising the liquid sheet between the end of the orifice and a section far downstream of the orifice, where viscosity has uniformized the velocity across the sheet and the pressure is equal to that of the air $(p=0$ in dimensionless variables), determines the axial velocity far downstream as $u_{\infty}=\left(\phi_{c m}+\phi_{p}\right) / \phi_{n}$, where $\phi_{m}=2 \pi \int_{r_{0}}^{1} u r \mathrm{~d} r, \phi_{c m}=2 \pi \int_{r_{0}}^{1} u^{2} r \mathrm{~d} r$ and $\phi_{p}=2 \pi \int_{r_{0}}^{1} p r \mathrm{~d} r$, with the integrals evaluated at the end of the orifice. Second, the equation for the kinetic energy of the liquid, $\nabla \cdot\left[\left(|v|^{2} / 2+p\right) v\right]=\nabla \cdot\left(\boldsymbol{v} \cdot \nabla \boldsymbol{\tau}^{\prime}\right)-\Phi_{v}$, where $\boldsymbol{\tau}^{\prime}$ is the viscous stress tensor and $\Phi_{v}$ is the viscous dissipation rate, can be integrated in the same control volume to give the energy balance $\left(u_{\infty}^{2}+v_{\infty}^{2}\right) \phi_{m} / 2=\phi_{H}+\Phi$, where $v_{\infty}$ is the radial velocity of the liquid far downstream of the orifice scaled here as the axial velocity, $\phi_{H}=2 \pi \int_{t_{0}}^{l}\left[\left(u^{2}+w^{2}\right) / 2+p\right] u r \mathrm{~d} r$, with the integral evaluated at the end of the orifice, and $\Phi$ is the viscous dissipation rate integrated over the control volume. If this quantity is neglected, the energy balance yields an approximate value of $v_{\infty}$, which, together with $u_{\approx}$ above, determines the half-angle of the cone in terms of magnitudes evaluated at the end of the orifice as

$$
\theta=\arccos \frac{\phi_{c m}+\phi_{p}}{\left(2 \phi_{m} \phi_{H}\right)^{1 / 2}} .
$$

The approximation (3.1) is similar to approximations proposed by Datta \& Som (2000), Yule \& Chinn (2000) and Nouri-Borujerdi \& Kebriaee (2012).

The approximate cone half-angle (3.1) is shown in figure $3(d)$ as a function of the atomizer constant. The approximation closely follows the inviscid prediction, for which there is no viscous dissipation, except for small values of the atomizer 

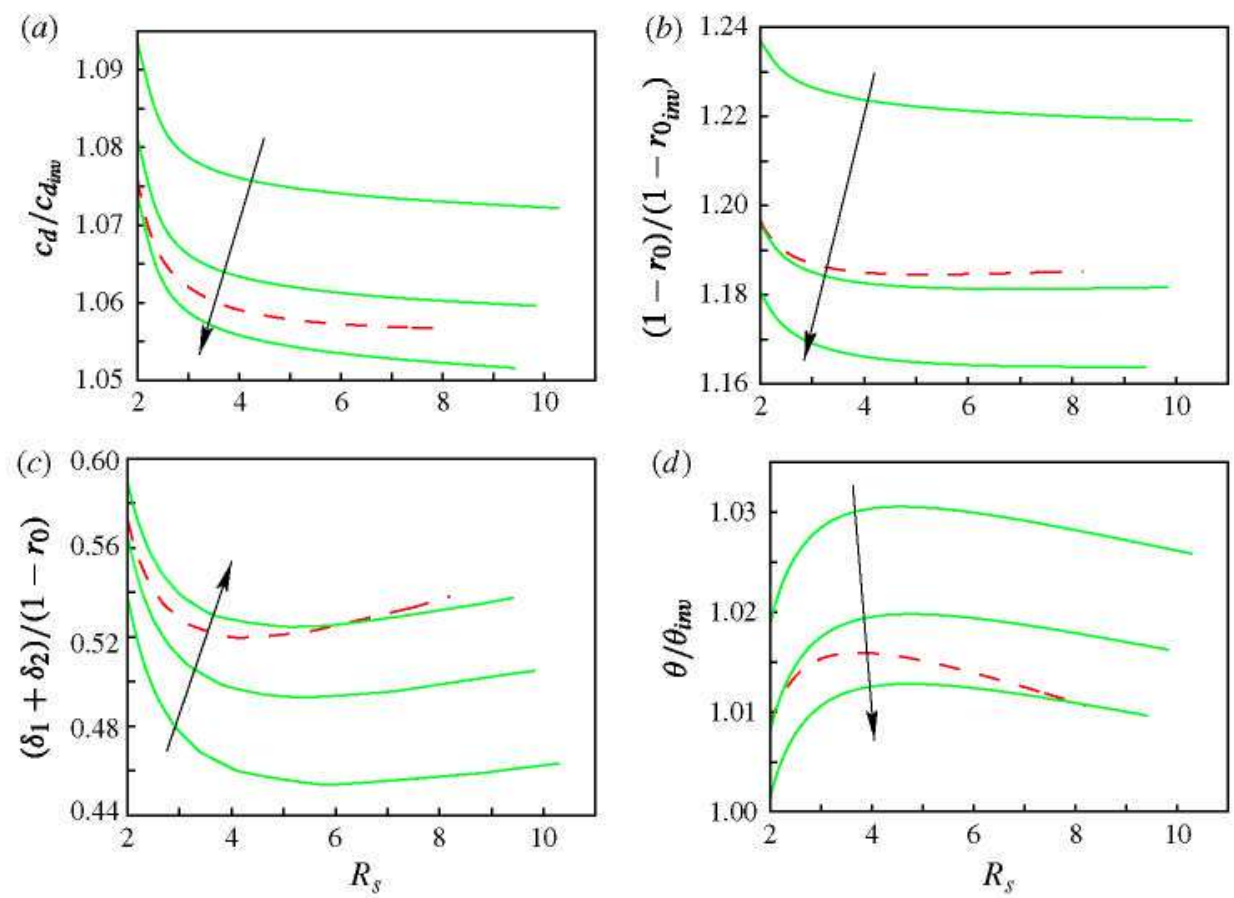

FIGURE 4. (Colour online) Atomizer characteristics as functions of the dimensionless radius of the chamber $R_{s}$ for $\ell_{1}=0$ and $\ell_{2}=0,0.2,0.4$, increasing as indicated by the arrows (solid curves), and for $\ell_{1}=0.5$ and $\ell_{2}=0.2$ (dashed curves). Values of other parameters are $R e=2000, \Delta=0.75, \epsilon=0.2, u_{c}=1$ and $\delta=0.245$. (a) Discharge coefficient scaled with its inviscid prediction $c_{d_{\text {in }}}$ given by $(2.1)$. (b) Liquid sheet thickness at the end of the orifice scaled with its inviscid prediction $1-r_{0_{i n v}}$ given by (2.1). (c) Sum of the boundary layer thicknesses at the end of the orifice scaled with the local thickness of the liquid sheet. $(d)$ Half-angle of the conical sheet scaled with its inviscid prediction $\theta_{i n v}$ given by Taylor (1948).

constant, where it falls below the inviscid prediction. This is an effect of the increasing thickness of the boundary layers of reduced azimuthal velocity when $\Delta$ decreases. Conservation of the Bernoulli constant in the inviscid flow, or of its cross-flow averaged value $\phi_{H} / \phi_{m}$ in the approximation above, roughly means that the azimuthal velocity at the end of the orifice becomes radial velocity farther downstream, causing the conical divergence of the sheet (Taylor 1948). The boundary layers of reduced azimuthal velocity tend to decrease $\phi_{H}$ and therefore the angle of the cone.

\subsection{Effects of other parameters}

The inviscid approximation predicts that the discharge coefficient, the thickness of the liquid sheet at the end of the orifice and the angle of the conical sheet far downstream of the orifice depend on the atomizer constant but are independent of the geometrical parameters $R_{s}, \ell_{1}$ and $\ell_{2}$. The computed values of these magnitudes scaled with their inviscid approximations are shown in figure 4 as functions of $R_{s}$ for various values of $\ell_{1}$ and $\ell_{2}$, with $R e=2000, \Delta=0.75(Q=3), \epsilon=0.2, u_{c}=1$ and $\delta=0.245$. The computed variations are small compared to the variations with $\Delta$ discussed above. This is in qualitative agreement with previous computations (Sakman 
et al. 2000) and experimental results (Dombrowski \& Hasson 1969; Rizk \& Lefebvre 1985; Suyari \& Lefebvre 1986), though the variations of $c_{d},\left(1-r_{0}\right)$ and $\theta$ in figure 4 are significantly smaller than the variations computed by Sakman et al. (2000) for $\Delta=0.3$. This is probably due to important differences in the internal flow, which could not be computed with the quasi-cylindrical approximation for the combination of large $\ell_{1}$ and small $\Delta$ used by these authors, as this combination seems to lead to centrifugal instability and extensive recirculation.

Inspection of the numerical solutions shows that some features of the flow do depend on the geometrical parameters, but they have only a weak effect on the displayed characteristics of the atomizer. Thus, owing to the manner in which the axial inlet velocity is defined in (2.9)-(2.11), the flux in the boundary layer around the air core increases when $R_{s}$ decreases. The streamwise evolution of this layer also depends on $R_{s}$. The axial velocity in the bulk of the chamber is not much smaller than the axial velocity in the boundary layer for the smallest values of $R_{s}$ in figure 4, so that both increase at about the same pace under the action of the pressure force required to keep the flow rate constant in the convergent chamber, and the thickness of the accelerated boundary layer decreases smoothly with streamwise distance. However, when $R_{s}$ increases, the axial velocity becomes much smaller in the bulk of the chamber than in the boundary layer. Then a small pressure force, which has little effect on the boundary layer, suffices to accelerate the slow flow in most of the chamber, while the largest pressure fall occurs around the orifice, where the thickness of the boundary layer decreases rapidly. It turns out that the thickness of the boundary layer at the end of the orifice, and the fraction of the flow rate that is in this layer, increase when $R_{s}$ decreases towards the minimum value shown in figure 4 . The thickness of the whole liquid sheet also increases (figure $4 b$ ), but less markedly than the thickness of the boundary layer, so that the ratio of the two thicknesses increases (figure $4 c$ ). Owing to the reduced swirling velocity in the boundary layer around the air core, the radial increase of the pressure across the liquid sheet at the end of the orifice decreases with $R_{s}$. This decreases the overpressure required in the chamber to drive the flow, and increases the discharge coefficient (figure $4 a$ ). The angle of the conical sheet also decreases with $R_{s}$ for small values of this parameter (figure $4 d$ ), owing to the increasing fraction of the flow that reaches the end of the orifice with a reduced swirling velocity. All the magnitude variations discussed here for small values of $R_{s}$ become smaller when the thickness of the boundary layer around the air core is decreased by decreasing $\delta$.

The boundary layer around the convergent wall of the chamber plays a role for large values of $R_{s}$. The thickness of this layer increases nearly linearly with $R_{s}$ (Binnie \& Harris 1950; Taylor 1950; Binnie et al. 1957; Burggraf et al. 1971; Bloor \& Ingham 1977), leading to the smooth increase at the right-hand side of figure $4(c)$ and to the decrease of the cone angle with increasing $R_{s}$ in figure $4(d)$.

The axial velocity of the flow in the bulk of the chamber decreases when $R_{s}$ increases, which makes the flow more sensitive to small pressure variations generated by the entrainment of the boundary layer at the convergent wall, where Ekman pumping leads to large velocities. Recirculation first appears at the shroud of this boundary layer when $R_{s}$ increases, as illustrated in figure 5 . The quasi-cylindrical approximation (2.2)-(2.5) can deal with a moderate recirculation, but eventually fails when the size of the recirculation region increases. This happens at the end points of the curves in figure 4 , which mark the limit beyond which the simplified formulation (2.2)-(2.12) cannot be used.

Figure 4 also show the effects of the dimensionless lengths of the orifice, $\ell_{2}$, and of the nearly cylindrical region of the chamber, $\ell_{1}$, on the characteristics of the 

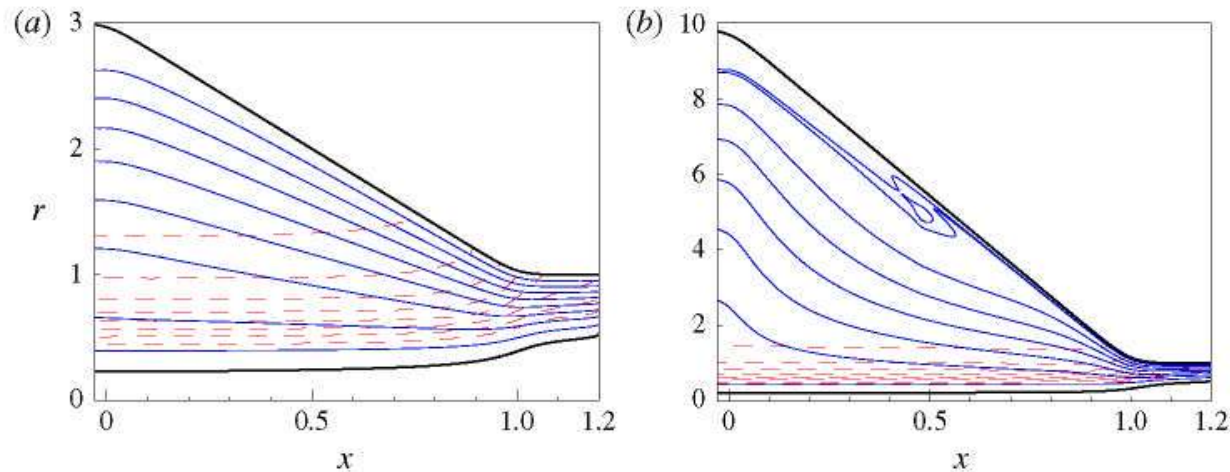

FIGURE 5. (Colour online) Streamlines (solid) and isobars (dashed) for $(a) R_{s}=3$ and (b) $R_{s}=9.84$. Values of other parameters are $R e=2000, \Delta=0.75, \ell_{1}=0, \ell_{2}=0.2, u_{c}=1$ and $\delta=0.245$.

atomizer. The effects of both parameters are moderate. The effect of $\ell_{2}$ (solid curves) can be understood by noting that the thickness of the boundary layer at the orifice wall increases with $\ell_{2}$; see figure $4(c)$. This increases the region of reduced swirl at the end of the orifice and leads to the decrease of the angle of the conical sheet shown in figure $4(d)$. The pressure in the chamber must increase to keep the flow rate constant when the boundary layer thickness and the friction force increase, leading to the reduction of the discharge coefficient seen in figure $4(a)$. The total thickness of the liquid sheet at the end of the orifice decreases when $\ell_{2}$ increases (figure $4 b$ ), despite the increase of the boundary layer thickness. This is an effect of the larger pressure force acting on the liquid outside the boundary layer, which increases the velocity at the end of the orifice. All these results are in qualitative agreement with the numerical results of Sakman et al. (2000) and references therein.

The thickness of the boundary layer of reduced swirl around the wall also increases, and the angle of the conical sheet decreases, with increasing $\ell_{1}$; compare the central solid curve and the dashed curve in figure $4(c, d)$. The discharge coefficient decreases slightly when $\ell_{1}$ increases from 0 to 0.5 , though this trend reverses for larger values of $\ell_{1}$ (results not displayed) in agreement with the results of Sakman et al. (2000). In any case, the increase of the boundary layer thickness at the end of the orifice now overcomes the small variation of the velocity outside the boundary layer and leads to the slight increase of the liquid sheet thickness with $\ell_{1}$ shown in figure $4(b)$.

Additional computations have been carried out varying the Reynolds number in the range 500-5000. In agreement with known experimental and numerical results (Dombrowski \& Hasson 1969; Jeng et al. 1998; Halder et al. 2003), these computations show that the thicknesses of the boundary layers at the end of the orifice increase when the Reynolds number decreases, but the effect of the Reynolds number on the discharge coefficient, the thickness of the liquid sheet and the angle of the conical sheet is small.

Wimmer \& Brenn (2013) pointed out that, at moderate liquid viscosities, for a given atomizer geometry and at a given driving pressure difference, the throughput of a liquid with higher viscosity is higher than that of a less viscous liquid. In dimensionless terms, when the driving pressure difference $\Delta p$ is used as a control variable instead of the flow rate, the viscosity of the liquid appears through the modified Reynolds number $\widetilde{R e}=\pi(2 \rho \Delta p)^{1 / 2} R_{0}^{2} /(L \mu)=4 R e \Delta / c_{d}$, and 
Wimmer \& Brenn's result amounts to saying that $c_{d}$ decreases with increasing $\widetilde{R e}$. To check that this result is reproduced by the quasi-cylindrical approximation, computations have been carried out in which $R e$ is varied keeping $R e \delta^{2}=12$ and other dimensionless parameters fixed at the values $\Delta=0.7, R_{s}=3, \ell_{1}=0$, $\ell_{2}=0.2$ and $u_{c}=1$. These computations give $c_{d}=(0.4468,0.4434,0.441,0.439)$ for $\widetilde{R e}=(14000,18000,22000,26000)$. Also in agreement with Wimmer \& Brenn's results, the radius of the air core at the end of the orifice increases, and the axial velocity at the liquid surface decreases, with increasing $\overparen{R e}$. However, the computed mean axial velocity, $c_{d} /\left(1-r_{0}^{2}\right)$, slightly increases with $\widehat{R e}$. Cousin \& Nuglish (2001) and Moon, Abo-Serie \& Bae (2010) note that the Reynolds number ceases to have an effect when it is increased to values well above those in our computations or in the work of Wimmer \& Brenn (2013).

\section{Conclusions}

A quasi-cylindrical approximation has been used to compute the axisymmetric flow of a viscous liquid and the radius of the air core in the chamber of a pressure swirl atomizer. Inlet conditions approximately accounting for the effect of the boundary layer at the wall of the chamber opposite to the exit orifice have been used, and an analysis of the critical flow at the end of the orifice has been carried out that gives a boundary condition at this end section.

Real flow effects on the characteristics of the atomizer have been analysed using as a reference the results of irrotational theory, which predicts that the discharge coefficient, the thickness of the liquid sheet at the end of the orifice and the angle of the conical sheet depend only on the atomizer constant. The numerical results show that the boundary layer around the air core makes the thickness of the liquid sheet larger than the inviscid prediction. However, Ekman pumping leads to large axial velocities in this boundary layer and in the boundary layer at the convergent wall of the chamber, increasing the discharge coefficient above the inviscid prediction. The radial increase of the pressure in the boundary layer around the air core is smaller than in an irrotational flow, owing to the reduced azimuthal velocity of the liquid in this region. The effect becomes more pronounced when the atomizer constant decreases, until the pressure increase becomes too small to steadily drive the flow towards the orifice when the atomizer constant becomes smaller than a certain value that depends on other parameters of the problem. An approximate expression for the angle of the conical sheet has been proposed that gives results in agreement with previous computations and closely follows the inviscid prediction except near the minimum value of the atomizer constant, when the presence of the boundary layers significantly reduces the outflow of angular momentum.

The effects of the chamber-to-orifice radius ratio and of the ratios of the length of the orifice to the length of the convergent region and of the length of the nearly cylindrical region to the length of the convergent region (to the extent that the latter ratio can be varied without exciting a centrifugal instability) on the characteristics of the atomizer are weaker than the effect of the atomizer constant. These effects can be largely explained in terms on the influence that the three parameters have on the boundary layers and their interaction with the flow in the bulk of the chamber.

\section{Acknowledgements}

This work was supported through projects DPI2010-20450-C03-01, DPI2013-47372C02-02 and CSD2010-00010 (Ministerio de Economía y Competitividad). 


\section{REFERENCES}

Abramovich. G. N. 1944 The theory of swirl atomizers. In Industrial Aerodynamics, pp. 114-121. BNT ZAGI (Central Aerodynamic Institute), Moscow.

Batchelor, G. K. 1967 An Introduction to Fluid Dynantics. Cambridge University Press.

BinNiE, A. M. 1949 The passage of a perfect fluid through a critical cross-section or "throat" Proc. R. Soc. Lond. A 197, 545-555.

BINNIE, A. M. \& HARRIS. D. P. 1950 The application of boundary-layer theory to swirling liquid flow through a nozzle. $Q$. J. Mech. Appl. Maths 3, 89-106.

Binnie, A. M. \& Hookings, G. A. 1948 Laboratory experiments on whirlpools. Proc. $R$. Soc. Lond. A 194, 398-415.

Binnie, A. M., Hookings, G, A, \& KAMEL, M. Y. M. 1957 The thow of swirling water through a convergent-diverging nozzle. $J$. Fluid Mech. 3, 261-274.

BLOOR, M. I. G. \& INGHAM. D. B. 1977 Axially symmetric boundary layers on a finite disk. Phys. Fluids 20. 1228-1233.

Burggraf, O. R., Stewartson, K. \& Belcher, R. 1971 Boundary layer induced by a potential vortex. Phys. Fluids 14, 1821-1833.

Chinn, J. J. 2009 An appraisal of swirl atomizer inviscid How analysis. Atomiz. Sprays 19. 263-311.

COOKE, J. C. 1952 On Pohlhausen's method with application to a swirl problem of Taylor. J. Aero. Sci. 19, $486-490$.

Cousin, J. \& Nuglish, H. J. 2001 Modeling of internal flow in high pressure pressure swurl injectors. SAE Paper 2001-01-0963.

DATTA, A. \& Som, S. K. 2000 Numerical prediction of air core diameter, coefficient of discharge and spray cone angle of a swirl spray pressure nozzle. Int $J$. Heat Fluid Flow 21, 412-419.

DOMBROWSKI. N. \& HASSON. D. 1969 The flow characteristics of swirl (centrifugal) spray pressure nozzles with low viscosity liquids. AIChE J. 15, 604-611.

Donjat, D., Estivalezes, J. L., Michau, M. \& Lavergne, G. 2003 Phenomenological study of the pressure swirl atomizer internal flow. In Proceedings of the $9 t h$ International Conference on Liquid Atomization and Spray Systems, Sorrento, Italy, pp, 12-19.

Dumouchel, C.. Bloor. M. I. G.. Dombrowski, N.. INGHaM. D. B. \& LedouX. M. 1992 Boundlary-layer characteristics of a swirl atomizer. Atomiz Sprays 2. 225-237.

GifFen, E. \& Muraszew, A. 1953 The Atomization of Liquid Fuels. Chapman and Hall.

HALDER, M. R., DASH, S. K.\& SOM, S. K. 2003 Influences of nozzle flow and nozzle geometry on the shape and size of an air core in a hollow cone swirl nozzle. Proc. Inst. Mech. Engrs 217, 207-217.

Hansen, K. G., Madsen. J.. Trinh. C. M., Ibsen, C. H. Solberg, T. \& Hiertager, B. H. 2002 A computational and experimental study of the internal flow in scaled pressure-swirl atomizer. In Proceedings of the 18th Annual Conference on Liquid Atomization and Spray Systems, Zaragoza, Spain, Paper 058.

Higuer A, F. J. 1994 The hydraulic jump in a viscous laminar flow. J. Fluid Mech. 274, 69-92.

Holtzclaw, D., Sakman, T., Jeng, S. M., Jog. M. A. \& Benjamin, M. A. 1997 Investigation of the flow in a simplex fuel nozzle. AIAA Paper 97-2970.

HORVAY, M. \& LEUCKEL. W. 1986 Experimental and theoretical investigation of swirl nozzles for pressure-jet atomization. Gennan Chem. Engng 9. 276-283.

JeNG, S. M.. Jog, M. A. \& Benjamin, M. A. 1998 Computational and experimental study of liquid sheet emanating from simplex fuel nozzle. AIAA J. 36, 201-207.

Kim, S., KhIL, T., Kim, D. \& Yoon, Y. 2009 Effect of geometric parameters on the liquid film thickness and air core formation in a swirl injector. Meas. Sci. Technol. 20, 1-11.

VON LAVAnTE. E. MaATJE, U. \& AlBina. F. A. 2002 Investigation of unstealy effects in pressure swirl atomizers. In Proceedings of the 18th Annual Conference on Liquid Atomization and Spray Systents, Zaragoza. Spain. Paper 081.

Lefebvre, A. H. 1989 Atomization and Sprays. CRC. Press.

MaAtJe, U., von Lavante, E. \& Albina, F. A. 2002 Numerical simulation of unsteady effects in simplex nozzles. AIAA Paper 2002-3179. 
Madsen, J.. HJertager, B. H. \& Solberg, T. 2002 Numerical simulation of internal flow in a large-scale pressure-swirl atomizer. In Proceedings of the 18th Annual Conference on Liquid Atomization and Spray Systems, Zaragoza, Spain, Paper 028.

MOON, S.. ABO-SERIE. E. \& BAE, C. 2010 Liquid film thickness inside the high pressure swirl injectors: real scale measurements and evaluation of analytical equations. Exp. Thern. Fluid Sci. 34, 113-121.

Nieuwkamp, W. C. 1985 Flow analysis of a hollow cone nozzle with potential flow theory. In Proceedings of the 1st Intemational Conference on Liquid Atomization and Spray Systems, London. $U K$, vol. IIIC/1, pp. 1-9.

Nouri-Borujerdi, A. \& KebriaeE. A. 2012 Numerical simulation of laminar and turbulent twophase flow in pressure-swirl atomizers. AlAA $J . \mathbf{5 0}, 2091-2101$.

NoviKov. I. I. 1948 Atomization of liquids by centrifugal nozzles. $J$. Tech. Phys. 18. 345-354.

PARK, H. \& Heister, S. D. 2006 Nonlinear simulation of free surfaces and atomization in pressure swirl atomizers. Phys. Fluids 18, 052103.

RizK, N. K. \& LefebvRe, A. H. 1985 Internal flow characteristics of simplex swirl atomizers. J. Propul. 1. 193-199.

Sakman. A. T. Jog. M. A., JENG, S. M. \& Benjamin. M. A. 2000 Parametric study of simplex fuel nozzle internal flow and performance. AIAA $J .38,1214-1218$.

Steinthorsson, E. \& LeE, D. M. 2000 Numerical simulations of internal flow in a simplex atomizer. In Proceedings of the 8th Intertational Conference on Liquid Atomization and Spray Systeins, Pasadena, CA, pp. 324-331.

SUYARI, M. \& LEFEBVRE. A. H. 1986 Film thickness measurements in a simplex swirl atomizer. J. Propul. 2. 528-533.

TAYLOR. G. I. 1948 The mechanics of swirl atomisers. In Proceedings of the 7 th International Congress of Applied Mechanics, vol. 2, pp. 280-285.

TAYLOR. G. I. 1950 The boundary layer in the converging nozzle of a swirl atomizer. $Q . J$. Mech. Appl. Maths 3, 129-139.

WANG, D., MA, Z.. JENG. S. M. \& BENJAMIN. M. A. 1999 Experimental study on large-scale simplex nozzle. AIAA Paper A99-31198.

WIMMER, E. \& BRENN, G. 2013 Viscous flow through the swirl chamber of a pressure-swirl atomizer. Intl J. Multiphase Flow' 53, 100-113.

Xue, J., Jog, M. A.. Jeng, S. M., Steinthorsson, E. \& Benjamin, M. A. 2004 Effect of geometric parameters on simplex atomizer performance. In Proceedings of the 35th Joint Propulsion Conference and Exhibit, Los Angeles, CA.

YULE, A. J. \& CHINN, J. J. 2000 The internal flow structure and exit conditions of pressure swirl atomizers. Atomiz, Sprays 10, 121-146.

Yule, A. J. \& WIDGER. I. R. 1996 Swirl atomizers operating at high water pressure. Ittl J. Mech. Sci. 38, 981-999. 